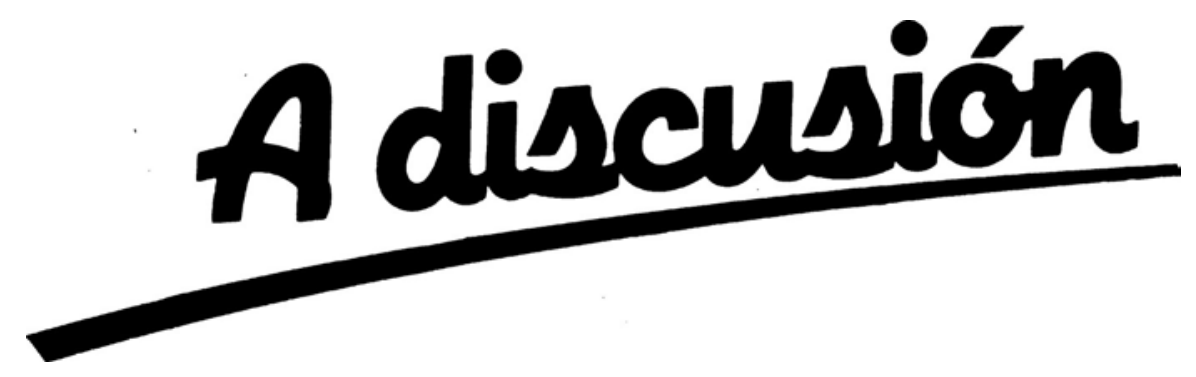

\title{
SELLING SHARES TO RETAIL INVESTORS: AUCTION VS. FIXED PRICE*
}

Jürgen Bierbaum and Veronika Grimm**

WP-AD 2004-08

Corresponding author: V. Grimm, Universidad de Alicante, Departamento de Fundamentos del Análisis Económico, Campus de San Vicente, 03071 Alicante, Spain. E-mail: grimm@merlin.fae.ua.es.

Editor: Instituto Valenciano de Investigaciones Económicas, S.A.

Primera Edición Febrero 2004.

Depósito Legal: V-1049-2004

IVIE working papers offer in advance the results of economic research under way in order to encourage a discussion process before sending them to scientific journals for their final publication.

\footnotetext{
* We thank Rabah Amir, Peter Bank, Ulrich Schmidt, and an anonymous referee, as well as seminar participants at Louvain la Neuve (CORE), Tilburg, Hannover, Stony Brook, Venice (ESEM), Berlin, and Alicante for helpful comments and suggestions. Financial support by the Deutsche Forschungsgemeinschaft, through SFB 373 ("Quantikation und Simulation "Okonomischer Prozesse"), Humboldt-Universität zu Berlin is gratefully acknowledged.

** J. Bierbaum: Humboldt-Universität Berlin, Institut für Wirtschaftstheorie I, Spandauer Straße 1, 10178 Berlin, Germany. V. Grimm: Departamento de Fundamentos del Análisis Económico, Universidad de Alicante, Campus de San Vicente, 03071 Alicante, Spain
} 


\title{
SELLING SHARES TO RETAIL INVESTORS: \\ AUCTION VS. FIXED PRICE
}

\author{
Jürgen Bierbaum and Veronika Grimm
}

\begin{abstract}
We analyze the problem of selling shares of a divisible good to a large number of buyers when demand is uncertain. We characterize equilibria of two popular mechanisms, a fixed price mechanism and a uniform price auction, and compare the revenues. While in the auction truthful bidding is a dominant strategy, we find that bidders have an incentive to overstate their demand in the fixed price mechanism. For some parameter values this yields the surprising result that the fixed price mechanism outperforms the auction.
\end{abstract}

Keywords: IPO, Uniform Price Auction, Open Offer, Proportional Rationing. JEL classification: D44, D45, G32 


\section{Introduction}

The three commonly used methods to sell shares in an initial public offering (IPO) are bookbuilding, open offer (or fixed price), and auctions. While bookbuilding and open offer have been predominant for a long time, auctions, recently held over the internet, are becoming more and more popular. It is still an open question which mechanism best serves the purposes of the seller. This is certainly due to the multitude of aspects to be taken into consideration.

Many papers on IPOs concentrate on informational aspects. Since the value of the issue is usually insufficiently known, the seller needs to gather information from large (informed) investors. Several authors show that bookbuilding allows to credibly extract information from large investors and thereby reduces the uncertainty about the issue. When compared to an auction, bookbuilding leads to more efficient information acquisition, since it gives the seller total discretion in the allocation of shares. ${ }^{1}$ To a lesser extent this also holds true for the fixed price method. ${ }^{2}$

While these informational aspects seem to be crucial in the relationship between seller and large investors, revenue is likely to be a predominant concern if it comes to selling shares to retail investors. Consider, for example, the following hybrid procedure which is indeed used in many countries: ${ }^{3}$ First, the seller allocates a proportion of shares to (informed) large investors

\footnotetext{
${ }^{1}$ Benveniste and Wilhelm (1989) show that with bookbuilding underpricing is necessary to effectively extract information from investors. Sherman and Titman (2000) analyze the bookbuilding method when information acquisition by large investors is costly. Moreover, Sherman (2000a) shows that bookbuilding leads to more efficient information acquisition than auctions.

${ }^{2}$ Benveniste and Wilhelm (1990) study a model with informed regular investors and uninformed retail investors. A combination of price and allocation discrimination between these groups leads to efficient revelation of the information held by informed investors. However, price discrimination is not allowed in some countries. Furthermore, there are fairness rules which regulate the allocation of shares.

${ }^{3}$ Sherman (2000a) provides a survey of IPO methods used by more than 40 countries.
} 
using a bookbuilding mechanism. Second, he sells the remaining shares to (uninformed) retail investors, using either a fixed price mechanism or an auction. Since information extraction is not the issue at this stage, the seller would like to choose the mechanism that maximizes expected revenue.

There are several reasons why selling shares to retail investors is important. To start with, it is a means of limiting the control of large investors over the firm. Furthermore, the issue may be too large to be absorbed by institutional investors only. Finally, the widespread availability of the internet makes the distribution of information by the seller and the placing of orders by retail investors cost-efficient and easy.

The aim of this paper is to compare the fixed price method and a uniform price auction when demand is uncertain (it can be either high or low) and there is a large number of potential buyers, who have private valuations. These assumptions seem to be justified if we consider the second stage of the mechanism described above, because it is reasonable to assume that the outcome of the bookbuilding stage will reveal the information held by institutional investors. Thus, after the bookbuilding stage, the common value of the issue is public information and demand of retail investors only depends on their preferences.

Usually, the fixed price method includes fairness rules which allow discrimination of buyers only on the basis of order size. In some countries, e. g. the United Kingdom, there is proportional (pro rata) rationing if the issue is oversubscribed. This motivates us to analyze the following variant of the fixed price method: First, the seller sets a price. Then, buyers submit their demand. If there is excess demand buyers are rationed according to the proportional rule.

Under proportional rationing with demand uncertainty bidders have an incentive to overstate their demand in order to alleviate the effects of being rationed in high demand scenarios. We show that the fixed price mechanism has a perfect Bayesian equilibrium, where bidders are rationed in the high demand scenario while there is no rationing in the low demand scenario. In 
equilibrium, buyers demand substantially more than their desired quantity.

Furthermore, we show that in a private values framework with a large number of small bidders truthful bidding a is dominant strategy in the uniform price auction. Thus, the auction yields the same revenue as if the seller was perfectly informed about demand but is restricted to linear prices. Therefore, it seems quite intuitive that the auction is the more profitable way to sell shares when demand is uncertain. As Sherman (2000a) puts it, "most finance academics would probably guess that auctions would be the best way to maximize the seller's revenues". To the best of our knowledge, however, this conjecture was never analyzed from a game-theoretic perspective.

The comparison of the two mechanisms yields a surprising result: Under certain parameter conditions the fixed price method outperforms the auction in terms of revenue. More precisely, this is the case when the return per share is relatively safe and aggregate demand is more likely to be low. Otherwise, the revenue in the fixed price mechanism is only slightly lower than the revenue in the auction. Moreover, since the variance of the payoff is lower in a fixed price mechanism ${ }^{4}$, a risk-averse seller would presumably prefer the fixed price method to the auction.

Uniform price auctions with a large number of bidders have been studied by Nautz (1995) for risk-neutral bidders and Nautz and Wolfstetter (1997) for risk-averse bidders. In both papers the authors argue that if there is a large number of bidders they act as price takers in a uniform price auction and therefore bid truthfully. Swinkels (2001) analyzes equilibria of uniform price auctions as the number of bidders tends to infinity and finds that the optimal strategy converges to truthful bidding.

Moulin (2000) and Herrero and Villar (2001) provide axiomatic analysis of rationing schemes. They show that proportional rationing is among the three rationing methods that satisfy "equal treatment of the equals", i. e. the allocation depends on bids only, and certain other procedural requirements.

\footnotetext{
${ }^{4}$ In many cases the revenue has zero variance at the price which maximizes expected revenue. This is shown in section 4.2.
} 
Moreover, the proportional rule is "fair", since the ratio of supply to demand is the same for all buyers. Finally, collusion of a group of buyers has no impact on the allocation to other buyers. The strategic incentives from rationing are analyzed, among others, by Bulow and Klemperer (1997) and Gilbert and Klemperer (2000). The first paper shows that prices which result in rationing can be optimal if a common value is to be sold, the second paper comes to the same conclusion for situations where customers must make sunk investments to enter the market. Nautz and Oechssler (2003) analyze proportional rationing in a private values framework, however, they obtain a nonexistence result that is due to the fact that bidders know that they are always rationed (there is no demand uncertainty).

This paper is organized as follows: In section 2 we present the model. Section 3 contains an analysis of the auction. In section 4 we present the fixed price mechanism. Then, we derive the equilibrium strategies of the buyers. Finally, we characterize the perfect Bayesian equilibrium of the entire game. Section 5 compares the expected revenue in the fixed price mechanism and the auction. Section 6 concludes. Proofs are relegated to the appendix unless they provide an intuition for the results.

\section{The Model}

One perfectly divisible unit of shares is to be sold by a seller whose objective is to maximize expected revenue. ${ }^{5}$ The final payoff per unit is a random variable $Y$ which is normally distributed with mean $\mu=1$ and variance $\sigma^{2}>0$.

There is a continuum $[\underline{\theta}, \bar{\theta}]$ of (potential) buyers, where $\underline{\theta}>0$. The Bernoulli utility function of buyer $\theta$ is given by

$$
u_{\theta}(w)=-\exp \left(-\frac{1}{\theta} w\right)
$$

\footnotetext{
${ }^{5}$ In Bierbaum and Grimm (2002) cases where the seller has different objectives are analyzed.
} 
where $w$ denotes the buyer's wealth, and $\theta^{-1}$ is the coefficient of absolute risk aversion of buyer $\theta$. The type $\theta$ is private information of each buyer and the distribution of buyers is given by a (probability) distribution function $F$. We assume that $F$ has no atoms, which implies that a single buyer has no weight, i. e. all buyers are small (retail) investors. The total mass of buyers is not perfectly known. Instead, there are two possible scenarios: with probability $g_{1}$ the mass is $m_{1}>0$ (scenario 1 ), with probability $\left(1-g_{1}\right)$ the mass is $m_{2}>m_{1}$ (scenario 2). Thus, the distribution of buyers in scenario $i$ is given by the cdf $^{6}$

$$
F_{i}=m_{i} F
$$

All this is common knowledge. Note that since the (probability) distribution $F$ over types is the same in each scenario, no buyer can infer the true distribution from his type.

Denote the initial wealth of buyer $\theta$ by $w_{\theta} \cdot{ }^{7}$ His expected utility of buying $\alpha$ shares at unit price $p$ is

$$
\begin{aligned}
U(\alpha, p, \theta) & =\mathbf{E}\left[-\exp \left(-\frac{1}{\theta}\left(\alpha(Y-p)+w_{\theta}\right)\right)\right] \\
& =-\exp \left(-\frac{w_{\theta}}{\theta}\right) \mathbf{E}\left[\exp \left(-\frac{1}{\theta} \alpha(Y-p)\right)\right] .
\end{aligned}
$$

Since $-\frac{1}{\theta} \alpha(Y-p) \sim N\left(-\frac{\alpha}{\theta}(\mu-p), \frac{\alpha^{2}}{\theta^{2}} \sigma^{2}\right)$ it holds that

$$
\mathbf{E}\left[\exp \left(-\frac{1}{\theta} \alpha(Y-p)\right)\right]=\exp \left(\frac{1}{2} \frac{\alpha^{2}}{\theta^{2}} \sigma^{2}-\frac{\alpha}{\theta}(\mu-p)\right) .
$$

The optimal number $\alpha$ of shares satisfies the following first-order condition:

$$
0=\left(\frac{\alpha}{\theta^{2}} \sigma^{2}-\frac{1}{\theta}(\mu-p)\right) \exp \left(\frac{1}{2} \frac{\alpha^{2}}{\theta^{2}} \sigma^{2}-\frac{\alpha}{\theta}(\mu-p)\right) .
$$

It follows that individual demand is

$$
\alpha(p, \theta)=\frac{\mu-p}{\sigma^{2}} \cdot \theta
$$

\footnotetext{
${ }^{6}$ Note that unless $m_{i}=1$ this is not a distribution function of a probability measure.

${ }^{7}$ Because of exponential utility the distribution of wealth does not affect our analysis.
} 
By integration we see that aggregate demand at price $p$ in scenario $i$ is

$$
A_{i}(p)=\int \alpha(p, \theta) d F_{i}(\theta)=\int\left(\frac{\mu-p}{\sigma^{2}} \cdot \theta\right) m_{i} d F(\theta)=\gamma_{i}(\mu-p),
$$

where

$$
\gamma_{i}=\frac{m_{i}}{\sigma^{2}} \int \theta d F(\theta)>0 .
$$

Since $\gamma_{2}>\gamma_{1}$ we can interpret scenario 1 and 2 as low demand scenario and high demand scenario, respectively. It follows from equation (5) that inverse demand in scenario $i$ is

$$
P_{i}(a)=\mu-\frac{a}{\gamma_{i}} .
$$

Recall that the seller can sell at most one unit. Therefore, the market clearing price in scenario $i$ is $P_{i}(1)=\mu-\frac{1}{\gamma_{i}}$, which is an increasing function of $\gamma_{i}$.

Now, we calculate the monopoly price $p_{i}$ in scenario $i$. Because of the capacity constraint a price below $P_{i}(1)$ cannot be optimal. Hence, the monopoly price solves

$$
\max _{p \in[0, \mu]} A_{i}(p) p \quad \text { s.t. } \quad p \geq P_{i}(1) .
$$

From the first-order condition $\gamma_{i}(\mu-2 p) \leq 0$ and the constraint $p \geq \mu-\frac{1}{\gamma_{i}}$ it follows that the monopoly price is

$$
p_{i}=\left\{\begin{array}{cl}
\frac{1}{2} \mu & \text { if } \mu<2 \frac{1}{\gamma_{i}}, \\
\mu-\frac{1}{\gamma_{i}} & \text { else. }
\end{array}\right.
$$

We assume that demand is so large that the capacity constraint is binding in both scenarios, i. e. $p_{i}=P_{i}(1)$ for $i=1,2$. This implies that $\gamma_{1} \geq 2 \mu^{-1}$.

\section{Uniform Price Auction}

When an auction is used for an IPO it is most often uniform-price sealedbid. ${ }^{8}$ This auction format meets various requirements like charging all customers an equal price ${ }^{9}$ and, in addition, is strategically simple when there

\footnotetext{
${ }^{8}$ See for example Kandel et al. (1999), who analyze IPO auctions in Israel, or Sherman (2000a).

${ }^{9} \mathrm{Cf}$. Benveniste and Wilhelm (1990).
} 
are many bidders. It is well known that there are quite unappealing low price equilibria when the number of bidders is small. However, if the number of bidders is large, tacit collusion may not be the issue as is shown in Biais and Faugeron-Crouzet (2000).

In order to analyze the auction game we have to distinguish the demand which is revealed in the auction from true aggregate demand $A_{i}$, as defined by equation (5).

Definition 1 (Revealed Demand) The revealed demand of bidder $\theta$ is a measurable and non-increasing function $p \mapsto d(p, \theta)$, where $0 \leq p \leq 1$. Aggregate revealed demand in scenario $i$ is given by

$$
D_{i}(p)=\int_{\underline{\theta}}^{\bar{\theta}} d(p, \theta) d F_{i}(\theta) .
$$

The rules of the auction are as follows: First, bidders submit their demand schedules. Thereafter, aggregate revealed demand is calculated. The auctioneer chooses the highest price such that aggregate revealed demand equals supply, i. e. in scenario $i$ the auction price is

$$
p_{i}^{A}=\max \left\{p \in[0,1]: D_{i}(p)=1\right\}
$$

Bidder $\theta$ obtains $d\left(p_{i}^{A}, \theta\right)$ shares at unit price $p_{i}^{A}$. If no market clearing price exists the seller keeps the shares and the bidders pay nothing.

The following lemma shows that if a market clearing price exists in scenario $i$ the revenue in the auction in this scenario is equal to the revenue which a monopolist would obtain from setting a linear price under complete information.

LEMMA 1 In any pure strategy equilibrium the auction price in scenario $i$ is $p_{i}^{A}=P_{i}(1)=p_{i}$. The revenue in the auction is $R_{i}^{A}=p_{i}$.

Proof Since there is continuum of buyers, a single buyer's bid has no impact on aggregate revealed demand. ${ }^{10}$ Therefore, bidders act as price

\footnotetext{
${ }^{10}$ It is well known that uniform price auctions create an incentive to reduce demand on
} 
takers. The continuum also implies that in any (pure strategy) equilibrium of the game there are only two equilibrium prices $p_{1}^{A}$ and $p_{2}^{A}$, which occur with positive probability. Thus, it is strictly dominant for buyer $\theta$ to demand quantity $\alpha\left(p_{i}^{A}, \theta\right)$ at price $p_{i}^{A}$. This implies that the equilibrium price in scenario $i$ is $p_{i}^{A}=P_{i}(1)=p_{i}$. Since supply is normalized to one, we also obtain $R_{i}^{A}=p_{i}$.

If we restrict our attention to equilibria with market clearing we obtain the following result, which immediately follows from the lemma.

THEOREM 1 The expected revenue in the auction is

$$
\pi_{A}=p_{1} g_{1}+p_{2}\left(1-g_{1}\right)=1-\frac{g_{1}}{\gamma_{1}}-\frac{1-g_{1}}{\gamma_{2}} .
$$

Quite intuitively, the expected revenue increases as the probability of the high demand scenario increases. As we have shown in lemma 1, the revenue in the auction is equal to the revenue that would be raised by linear monopoly prices under complete information about the demand scenario. Note that this implies that setting a reservation price does not improve the auction. Moreover, the auction is strategically very simple for both, the seller and the buyers. None of them needs to take into consideration any information except for their own in order to derive their optimal strategies. These properties make the auction a very appealing selling mechanism.

\section{$4 \quad$ Fixed Price plus Proportional Rationing}

In this section we describe and analyze the second mechanism: The seller sets a fixed price and applies proportional rationing in the case of excess demand. Proportional rationing is used in several countries and seems to all but the first unit if bidders may influence the price by their bid. See, for example, Wilson (1979) and Back and Zender (1993). However, for a continuum of buyers a single buyer has no impact on aggregate revealed demand. This is similar to the results of Nautz and Wolfstetter (1997) and Swinkels (2001), who analyze auctions when there is a large number of bidders. 
be a good approximation for other rationing methods applied in IPOs, since it satisfies certain fairness rules. ${ }^{11}$ While priority rules may also be used to allocate shares to specific groups of investors, proportional rationing is most likely being applied within these groups.

Definition 2 (Proportional Rationing) Let $d \geq 0$ be a buyer's individual revealed demand, $D>0$ aggregate revealed demand, and denote total supply by $S>0$. Under proportional rationing the buyer gets

$$
d^{s}=\min \left(d, d \frac{S}{D}\right)=d \cdot Q(S, D)
$$

where

$$
Q(S, D)=\min \left(1, \frac{S}{D}\right)
$$

is called rationing factor.

Recall that in our model the seller can sell at most one unit. Thus, bidders are rationed whenever aggregate revealed demand exceeds 1 . However, revealed demand may also fall short of 1 , in which case each bidder gets the demanded quantity.

Note that under proportional rationing truthful bidding cannot be an equilibrium. To see this, suppose that all the other bidders bid truthfully. Then, a bidder has an incentive to overbid his true demand in order to increase the (too low) quantity he gets in the high demand scenario at the (initially low) cost of getting a bit too much in case demand is low. Since this increases demand in the low demand scenario, the seller's expected payoff is certainly higher than under truthful bidding and the market clears at prices strictly higher than $p_{1}$.

The above reasoning raises two questions: First, does the fixed price mechanism have an equilibrium at all ${ }^{12}$ and, if so, can revealed demand be

\footnotetext{
${ }^{11}$ For a detailed analysis of the properties of proportional rationing and other rationing rules cf. Moulin (2000) or Herrero and Villar (2001).

${ }^{12}$ This is also of practical relevance, since it is not very appealing to use a selling mechanism that has no equilibrium. Nautz and Oechssler (2003) showed that there is no equilibrium of the proportional rationing game when demand is certain.
} 
high enough to allow for charging a price that yields a higher expected payoff than the auction?

In order to be able to compare the expected revenues in the auction and the fixed price mechanism, in this section we first characterize the equilibrium of the fixed price mechanism. We start with the description of the game.

First, nature draws the state of the world (i. e. scenario 1 or scenario 2). Neither the buyers, nor the seller observe the state, however, it is common knowledge that the probability of low demand is $g_{1}$. Thereafter, we have two stages. At the first stage, the seller offers one unit of shares at a price $p \geq 0$ and decides on an upper limit on individual demand, $\bar{d}$ (i. e. no single buyer can ask for more than $\bar{d}$ shares). At the second stage, which we call bidding stage, each buyer submits his individual demand $0 \leq d \leq \bar{d}$ as a sealed bid. From the bids aggregate revealed demand $D$ is calculated. Finally, a buyer who has demanded $d$ shares gets $d^{s}$ shares and pays $d^{s} p$, where $d^{s}$ is determined by proportional rationing.

As in section 3, denote buyer $\theta^{\prime} s$ revealed demand at price $p$ by $d(p, \theta)$. It follows that revealed demand $D_{i}(p)$ in scenario $i$ is given by (10). For simplicity we denote the corresponding rationing factor by

$$
Q_{i}=Q\left(1, D_{i}(p)\right)=\min \left(1, \frac{1}{D_{i}(p)}\right)
$$

Since the true scenario is unknown, the rationing factor is a random variable $\widetilde{Q}$ with realization $Q_{i}$ in scenario $i$.

Now, we can determine the players' payoffs. The seller's revenue in scenario $i$ is given by

$$
R_{i}^{F}(p)=Q_{i} D_{i}(p) \cdot p
$$

Hence, expected revenue is

$$
\pi_{F}(p)=R_{1}^{F}(p) g_{1}+R_{2}^{F}(p)\left(1-g_{1}\right)
$$

Since it is common knowledge that the buyers have the same information about the demand scenario as the seller, the price set by the seller at stage 
one cannot reveal information about the demand scenario. ${ }^{13}$ Therefore, for a given rationing factor $\widetilde{Q}$ the expected utility of buyer $\theta$ under proportional rationing is

$$
\begin{aligned}
\mathbf{E}_{g_{1}}[U(d(p, \theta) \widetilde{Q}, p, \theta)]= & U\left(d(p, \theta) Q_{1}, p, \theta\right) g_{1} \\
& +U\left(d(p, \theta) Q_{2}, p, \theta\right)\left(1-g_{1}\right) .
\end{aligned}
$$

Note that the expected utility of buyer $\theta$ depends only on the aggregate numbers $Q_{1}$ and $Q_{2}$ while individual demand of other buyers plays no role. ${ }^{14}$

\subsection{Optimal Bidding in the Rationing Game}

In this section we analyze the bidding stage that starts after a price $p \in$ $\left[p_{1}, p_{2}\right]$ has been announced by the seller. ${ }^{15}$ Note that for a given price $p$ and a given belief about the probability of the low demand scenario the set of sequentially rational strategies of the buyers coincides with the set of Nash equilibria of the game that corresponds to the bidding stage. Thus, although the bidding stage is no (sub-) game in a strict sense we speak of an equilibrium of the bidding stage. We proceed in two steps: First, we analyze a bidder's best reply against a given random rationing factor, and thereafter we use the result in order to characterize the equilibrium of the bidding stage. In this section we ignore the upper limit on revealed demand $\bar{d}$, since the analysis would only be complicated without providing additional insights. As we will show in section 4.2, the seller never chooses an upper limit that affects bidding behavior at the price he chooses in equilibrium. ${ }^{16}$

Let us start by deriving the best response (optimal demand) of a single buyer $\theta$ at price $p$ for a given aggregate revealed demand of the other buyers.

\footnotetext{
${ }^{13}$ See also condition B(iii), "no signaling what you don't know", in Fudenberg and Tirole's (1998) definition of perfect Bayesian equilibrium.

${ }^{14}$ The bidding stage resembles an anonymous game as analyzed by Blonski (2001).

${ }^{15}$ It is easy to show that in equilibrium no price outside this interval will be posted by a seller.

${ }^{16}$ However, $\bar{d}$ will be crucial in order to be able to establish existence of an equilibrium of the fixed price mechanism.
} 
Since there is a continuum of buyers, a single buyer's demand $d$ has no impact on aggregate revealed demand $D_{i}$ in scenario $i$. We assume that $D_{1}<D_{2}$ which implies $Q_{1}>Q_{2} \cdot{ }^{17}$

A buyer's best response to a given rationing factor $\widetilde{Q}$ is a solution to the following optimization problem:

$$
\max _{d \geq 0} \mathbf{E}_{g_{1}}[U(d \widetilde{Q}, p, \theta)] .
$$

The first-order condition of buyer $\theta$ 's optimization problem is ${ }^{18}$

$$
0=\mathbf{E}_{g_{1}}\left[\left(\frac{d}{\theta^{2}} \sigma^{2} \widetilde{Q}^{2}-\frac{1}{\theta}(\mu-p) \widetilde{Q}\right) \exp \left(\frac{1}{2} \frac{d^{2}}{\theta^{2}} \sigma^{2} \widetilde{Q}^{2}-\frac{d}{\theta}(\mu-p) \widetilde{Q}\right)\right] .
$$

Since $\alpha(p, \theta)>0$, we can decompose $d=x \cdot \alpha(p, \theta)$, where $x$ represents the relative markup over the optimal number of shares.

Recall that under proportional rationing the quantity a buyer gets in scenario $i$ is $d_{i}^{s}=x \alpha(p, \theta) Q_{i}$. Obviously, a choice of $x$, where $x \alpha(p, \theta) Q_{1}<$ $\alpha(p, \theta)$ cannot be optimal which implies $x \geq Q_{1}^{-1}$. It follows from a similar argument that $x \leq Q_{2}^{-1}$. To choose the optimal $x$ from the interval $\left[Q_{1}^{-1}, Q_{2}^{-1}\right]$ the buyer has to consider the trade-off between getting too little shares in the case of high demand and getting too many shares in the case of low demand.

In the following, we will show that there is a unique solution to the buyer's problem, and that the markup factor that solves the above mentioned trade off optimally does not depend on the buyer's type. Substituting $x \alpha(p, \theta)$ for $d$ in the first-order condition and multiplying by $x$ yields

$$
\begin{aligned}
0 & =\mathbf{E}_{g_{1}}\left[\left(x^{2} \widetilde{Q}^{2}-x \widetilde{Q}\right) \exp \left(\frac{1}{2}\left(\frac{\mu-p}{\sigma}\right)^{2} x^{2} \widetilde{Q}^{2}-\left(\frac{\mu-p}{\sigma}\right)^{2} x \widetilde{Q}\right)\right] \\
& =\mathbf{E}_{g_{1}}\left[h_{p}(x \widetilde{Q})\right]
\end{aligned}
$$

where $h_{p}$ is defined by

$$
h_{p}(y)=\left(y^{2}-y\right) \exp \left(\left(\frac{\mu-p}{\sigma}\right)^{2}\left(\frac{1}{2} y^{2}-y\right)\right) .
$$

\footnotetext{
${ }^{17}$ We will later show that this inequality holds in equilibrium.

${ }^{18}$ Here, we used that the conditional distribution of $\widetilde{Q}(Y-p)$ given scenario $i$ is $N\left(Q_{i}(\mu-p), Q_{i}^{2} \sigma^{2}\right)$.
} 
It is shown in the appendix that a unique solution $x_{\widetilde{Q}}\left(p, g_{1}\right) \in\left(Q_{1}^{-1}, Q_{2}^{-1}\right)$ to $(20)$ exists. Moreover, since $\mathbf{E}_{g_{1}}\left[h_{p}(x \widetilde{Q})\right]$ does not depend on $\theta$, the markup factor is type-independent. Inserting the optimal markup factor $x_{\widetilde{Q}}\left(p, g_{1}\right)$ into the decomposition $d=x \cdot \alpha(p, \theta)$ yields the following lemma which summarizes the above results:

LEMmA 2 There is a unique revealed demand $d_{\widetilde{Q}}\left(p, \theta, g_{1}\right)=x_{\widetilde{Q}}\left(p, g_{1}\right) \alpha(p, \theta)$ which maximizes the utility of buyer $\theta$ for a given rationing factor $\widetilde{Q}$. The markup factor $x_{\widetilde{Q}}\left(p, g_{1}\right)$ does not depend on the buyer's type $\theta$. Moreover, it holds that $x_{\widetilde{Q}}\left(p, g_{1}\right) \in\left(Q_{1}^{-1}, Q_{2}^{-1}\right)$.

Now, we can characterize the equilibrium of the bidding stage. In equilibrium each bidder chooses his best response revealed demand as characterized in lemma 2 and, at the same time, the rationing factor must result from the aggregate demand revealed by the bidders. Thus, if $D_{i}(p)$ is the realization of equilibrium aggregate demand $\widetilde{D}$ in scenario $i$ it must hold that

$$
D_{i}(p)=\int_{\underline{\theta}}^{\bar{\theta}} x_{\widetilde{Q}}\left(p, g_{1}\right) \alpha(p, \theta) d F_{i}(\theta)=x_{\widetilde{Q}}\left(p, g_{1}\right) A_{i}(p),
$$

where $\widetilde{Q}$ is the rationing factor corresponding to $\widetilde{D}$. It follows that an equilibrium of the bidding stage is described by a common markup factor $x=x\left(p, g_{1}\right)$ where $\widetilde{Q}$ is given by its realizations

$$
Q_{i}=\min \left(1, \frac{1}{x A_{i}(p)}\right), \quad i=1,2
$$

In order to emphasize that $\widetilde{Q}$ is the rationing factor which corresponds to $x$ we write $\widetilde{Q}_{x}$. From identity (22) it follows that $\frac{D_{1}}{D_{2}}=\frac{A_{1}}{A_{2}}$ in equilibrium. Thus, revealed demand in scenario 2 is larger than revealed demand in scenario 1 , i. e. scenario 2 is the high demand scenario, as asserted. Inserting $Q_{1}=1$ and $Q_{2}=\left(x A_{2}(p)\right)^{-1}$ into $(20)$ yields that the equilibrium markup factor 
solves

$$
\begin{aligned}
0= & \left(x^{2}-x\right) \exp \left(\left(\frac{\mu-p}{\sigma}\right)^{2}\left(\frac{1}{2} x^{2}-x\right)\right) g_{1} \\
& +\left(A_{2}^{-2}-A_{2}^{-1}\right) \exp \left(\left(\frac{\mu-p}{\sigma}\right)^{2}\left(\frac{1}{2} A_{2}^{-2}-A_{2}^{-1}\right)\right)\left(1-g_{1}\right) .
\end{aligned}
$$

The following theorem shows that a unique equilibrium (in pure strategies) of the bidding stage exists if the price is higher than a critical price that depends on the probability of the low demand scenario, $g_{1}$.

ThEOREM 2 Let $p \in\left[p_{1}, p_{2}\right]$ and let $g_{1}$ be the probability of the low demand scenario. The following claims hold true:

(i) There is a unique price $p_{e}=p_{e}\left(g_{1}\right) \in\left[p_{1}, p_{2}\right]$ such that an equilibrium of the bidding stage exists if and only if $p \geq p_{e}$. The function $g_{1} \mapsto p_{e}\left(g_{1}\right)$ is strictly decreasing, with $p_{e}(0)=p_{2}$ and $p_{e}(1)=p_{1}$.

(ii) For $p>p_{e}$ the equilibrium is unique. In this case, the equilibrium markup factor $x\left(p, g_{1}\right)$ satisfies $x\left(p, g_{1}\right)<A_{1}(p)^{-1}$.

(iii) The only market clearing price is $p_{e}$, i. e. $D_{i}\left(p_{e}\right) \geq 1$ for $i=1,2$. It holds that $x\left(p_{e}, g_{1}\right) \geq A_{1}\left(p_{e}\right)^{-1}$.

Proof See the appendix.

By combining lemma 2 and theorem 2 we obtain the following corollary:

Corollary 1 Let $p \in\left(p_{e}, p_{2}\right)$ and $g_{1} \in(0,1)$. In the equilibrium of the bidding stage there is no rationing in scenario 1 while all buyers are rationed in scenario 2. In particular, it holds that the supply to buyer $\theta$ is

$$
d^{s}(p, \theta)= \begin{cases}x\left(p, g_{1}\right) \alpha(p, \theta) & \text { in scenario } 1, \\ A_{2}(p)^{-1} \alpha(p, \theta) & \text { in scenario } 2 .\end{cases}
$$


Proof See the appendix.

The corollary shows that in equilibrium the number of shares which a buyer gets in the high demand scenario does not depend on $x$ while the number of shares in the low demand scenario exceeds true demand by a factor of $x$. Therefore, the markup factor $x$ is optimally chosen such that a marginal increase of the bid would increase the buyer's utility in scenario 2 by the same amount as it would decrease his utility in scenario 1. In equilibrium the quantity a buyer gets in scenario 2 is given by $\alpha(p, \theta) / A_{2}$ and therefore marginal utility in the high demand scenario is fixed. If $p$ is too small relative to $g_{1}$, marginal utility at $\alpha(p, \theta) / A_{2}$ is higher than marginal disutility at $x \alpha(p, \theta)$ for all $x$, where aggregate revealed demand in scenario $1, x A_{1}$, is lower than one. This provides an incentive to overstate demand in a way that bidders are rationed in both scenarios. If this is the case, it is costless to increasingly exaggerate bids and, thus, no equilibrium exists if $g_{1}$ is too high relative to $p$.

REMARK 1 (No TRADE) It can be shown that with exponential utility and proportional rationing there is no incentive for aftermarket trade. At the final allocation the willingness to pay for an additional unit is independent of the buyer's type.

\subsection{Equilibrium of the Fixed Price Mechanism}

Now that we have characterized the equilibrium of the second stage of the game, we are prepared to characterize the equilibrium of the fixed price mechanism. The seller's objective is to choose $p$ and $\bar{d}$ as to maximize expected revenue, anticipating the choice of the equilibrium markup factor at the second stage.

If we ignore the upper limit on revealed demand, the seller's revenue $R_{i}^{F}$ in scenario $i$ at a price $p \in\left[p_{e}\left(g_{1}\right), p_{2}\right]$ is given by

$$
R_{1}^{F}(p)=\left\{\begin{array}{cl}
p & \text { if } p=p_{e}\left(g_{1}\right) \\
x\left(p, g_{1}(p)\right) A_{1}(p) p & \text { if } p>p_{e}\left(g_{1}\right)
\end{array}\right.
$$


and

$$
R_{2}^{F}(p)=p
$$

respectively. Hence, the expected revenue is

$$
\pi_{F}(p)=R_{1}^{F}(p) g_{1}+R_{2}^{F}(p)\left(1-g_{1}\right)
$$

It is straightforward to show that $\pi_{F}$ is a continuous function.

Note that at $p=p_{e}\left(g_{1}\right)$ the seller sells the whole quantity in both scenarios. Therefore, in equilibrium, he will never post a price below $p_{e}\left(g_{1}\right)$. Furthermore, if the upper limit $\bar{d}$ is binding for some or all bidders at a price $p \in\left[p_{e}\left(g_{1}\right), p_{2}\right]$, this unambiguously reduces the seller's expected revenue at that price $p$. Therefore, in equilibrium the seller will choose $\bar{d}$ high enough not to affect revealed demand in equilibrium. Thus, the upper limit does not affect the equilibrium outcome. However, it ensures existence of an equilibrium at the second stage also at prices below $p_{e}\left(g_{1}\right)$. We are now prepared to state the main theorem of this section:

Theorem 3 A perfect Bayesian equilibrium (in pure strategies) of the fixed price mechanism exists. Every equilibrium strategy profile $\left(p^{*}, \bar{d}^{*},\left(d^{*}(p, \theta)_{p}\right)_{\theta}\right)$ satisfies

$$
\begin{gathered}
p^{*} \in\left[p_{e}\left(g_{1}\right), p_{2}\right], \\
\bar{d}^{*} \geq x\left(p^{*}, g_{1}\right) \alpha\left(p^{*}, \bar{\theta}\right), \\
d^{*}(p, \theta)=x\left(p, g_{1}\right) \cdot \alpha(p, \theta) \quad \text { for } \quad p \in\left[p^{*}, p_{2}\right], \\
\pi_{R}\left(p^{*}\right) \geq p_{e}\left(g_{1}\right) .
\end{gathered}
$$

ProOF If the upper limit $\bar{d}$ is high enough not to affect revealed demand at prices in $\left[p_{e}\left(g_{1}\right), p_{2}\right]$ equilibrium demand of buyer $\theta$ at price $p \in\left[p_{e}\left(g_{1}\right), p_{2}\right]$ is given by

$$
d^{*}(p, \theta)=x\left(p, g_{1}\right) \cdot \alpha(p, \theta) .
$$

At price $p_{e}\left(g_{1}\right)$ all shares are sold in both scenarios which implies that expected profit is save and equal to $p_{e}\left(g_{1}\right)$. Since there is only one unit for sale, 
setting a price below $p_{e}$ is strictly dominated for the seller. The expected revenue $\pi_{F}$ is continuous on the compact set $\left[p_{e}\left(g_{1}\right), p_{2}\right]$. Thus, there exists an equilibrium price $p^{*} \in\left[p_{e}\left(g_{1}\right), p_{2}\right]$ that maximizes the seller's revenue.

Since $\bar{d}$ can only reduce the demanded quantity, the seller strictly prefers a limit that does not affect revealed demand by any bidder at the optimal price $p^{*}$, i. e. $\bar{d}^{*} \geq x\left(p^{*}, g_{1}\right) \alpha\left(p^{*}, \bar{\theta}\right)$. We show in the appendix that due to the upper bound on revealed demand an equilibrium of the bidding stage exists at all prices $p \in\left[p_{1}, p_{2}\right]$.

The theorem shows that the equilibrium payoff $\pi_{F}\left(p^{*}\right)$ is uniquely determined. It includes two possible cases: $p^{*}=p_{e}\left(g_{1}\right)$ and $p^{*}>p_{e}\left(g_{1}\right)$. By theorem 2 the seller realizes a riskless profit equal to $p_{e}\left(g_{1}\right)$ in the first case while in the second case the return is risky, with $R_{2}^{F}\left(p^{*}\right)=p^{*}>R_{1}^{F}\left(p^{*}\right)$. We have run numerical simulations which show that both cases can occur depending on the choice of the parameters $\sigma, \gamma_{1}, \gamma_{2}$ and $g_{1} \cdot{ }^{19}$

\subsection{Comparative Statics}

In this section we study the comparative statics properties of the equilibrium markup factor $x\left(p, g_{1}\right)$ at a price $p>p_{e}\left(g_{1}\right)$ with respect to $\sigma^{2}$.

In order to characterize the effect of the asset's risk on the equilibrium markup factor we fix $p \in\left(p_{e}\left(g_{1}\right), p_{2}\right)$ and $\gamma_{i}(i=1,2) .{ }^{20}$ That is, we assume that aggregate demand $A_{i}(p)$ remains constant while $\sigma^{2}$ varies. By definition of $\gamma_{i}$ this implies that mean risk aversion in the population decreases as $\sigma^{2}$ increases.

Consider the function $c(p)=\left(\frac{\mu-p}{\sigma}\right)^{2}$, which obviously is decreasing in $\sigma^{2}$. Therefore, an increase in $\sigma^{2}$ is equivalent to a decrease in $c$.

Consider the indirect utility function $V(z)=U(z \cdot \alpha(p, \theta), p, \theta)$ of buyer $\theta$. It holds that

$$
V(z)=-\exp \left(\frac{c}{2}(z-1)^{2}\right) \exp \left(-\frac{w_{\theta}}{\theta}-\frac{c}{2}\right) .
$$

\footnotetext{
${ }^{19} \mathrm{Cf}$. Bierbaum and Grimm (2002).

${ }^{20}$ Note that $p_{e}$ depends on $\sigma^{2}$. However, if $p>p_{e}\left(g_{1}\right)$ for some $\sigma^{2}$ this inequality also holds true for every $\widetilde{\sigma}^{2}$ close to $\sigma^{2}$.
} 
Recall that the equilibrium markup factor $x$ solves an optimal trade-off between the two demand scenarios, i. e. the expected marginal benefit of overstating demand in scenario 2 equals the expected marginal loss of doing so in scenario 1. In equilibrium, the marginal utilities of buyer $\theta$ are $V^{\prime}\left(A_{2}^{-1}\right)$ in scenario 2 and $V^{\prime}(x)$ in scenario 1, respectively. Now, as $c$ varies the equilibrium quantity in scenario 2 does not change, since $A_{2}$ remains constant. However, since $c$ determines the marginal utilities in scenario 1 and 2 , $x$ depends on $c$. In the following, we characterize this dependence of $x$ on $c$.

First, we rewrite the equilibrium condition (24) as

$$
\begin{aligned}
\frac{x}{A_{2}^{-1}} \cdot \frac{g_{1}}{1-g_{1}} & =\frac{-\left(A_{2}^{-1}-1\right) \exp \left(\frac{c}{2}\left(A_{2}^{-1}-1\right)^{2}\right)}{(x-1) \exp \left(\frac{c}{2}(x-1)^{2}\right)} \\
& =\frac{-V^{\prime}\left(A_{2}^{-1}\right)}{V^{\prime}(x)} .
\end{aligned}
$$

To analyze the behaviour of (29) with respect to changes in $c$ we define

$$
\Delta=\left(A_{2}^{-1}-1\right)^{2}-(x-1)^{2} .
$$

For $\Delta>0$ the RHS of (29) is strictly increasing in $c$, while for $\Delta<0$ it is strictly decreasing. For $\Delta=0$ the RHS is constant in $c$. Since $c>0$ and $x \geq 1$, the RHS is strictly decreasing in $x$ while the LHS is strictly increasing. It follows that in the case $\Delta>0$ an increase in $c$ has to be compensated by an increase in $x$ which, in turn, decreases $\Delta$. For $\Delta<0$ it is the other way around.

These findings are summarized in the following proposition:

Proposition 1 Let $x$ be the equilibrium markup factor at $p$, for parameters $g_{1}$ and $\sigma^{2}$.

(i) If $\Delta>0$ the equilibrium markup factor $x$ is strictly decreasing in $\sigma^{2}$.

(ii) If $\Delta=0$ the equilibrium markup factor $x$ is constant in $\sigma^{2}$.

(iii) If $\Delta<0$ the equilibrium markup factor $x$ is strictly increasing in $\sigma^{2}$. 


\section{Auction versus Fixed Price}

Now we are prepared to compare the revenues of the two mechanisms. In the fixed price mechanism, as we have shown in the previous section, bidders have an incentive to overstate their demand under proportional rationing, which raises the expected revenue at a given price. However, because of the fixed price, this mechanism does not adapt to the realized scenario. In the auction, prices optimally adapt to the realized scenario while for a given price demand is lower than in the fixed price mechanism. Thus, we have to find out which of these properties is the more important one. We will show that the answer to this question is ambiguous and depends on the parameters of the model.

It seems impossible to derive the expected profit in the fixed price method explicitly. Instead, we use an indirect approach, where we compare the expected revenues in the auction and the fixed price mechanism with a benchmark revenue. The benchmark revenue is the expected revenue which would obtain if buyers bid as if they were risk-neutral when choosing the markup factor under proportional rationing. We prove in lemma 3 that the benchmark revenue and the expected revenue in the auction are equal. Then, in lemma 4, we compare the markup factor in the fixed price mechanism with the markup factor in the benchmark case. Finally, we use these lemmas to obtain a comparison of the auction and the fixed price mechanism (theorem $4)$.

If a buyer is risk-neutral with respect to the choice of the markup factor

$x$ he maximizes his utility given the expected value $\hat{Q}_{x}=\mathbf{E}_{g_{1}}\left[\widetilde{Q}_{x}\right]$ of $\widetilde{Q}_{x}$, as defined in section 4.2. The first-order condition (20) becomes

$$
\left(x^{2} \hat{Q}_{x}^{2}-x \hat{Q}_{x}\right) \exp \left(\left(\frac{\mu-p}{\sigma}\right)^{2}\left(\frac{1}{2} x^{2} \hat{Q}_{x}^{2}-x \hat{Q}_{x}\right)\right)=0 .
$$

Thus, the benchmark markup factor solves $x=\hat{Q}_{x}^{-1}$ which ensures that buyer $\theta$ gets $\alpha(p, \theta)$ shares in expectation.

Since there is only one unit for sale, the fixed price mechanism cannot 
raise a higher expected revenue than the auction if the price is below $\pi_{A}$. Thus, we can restrict our analysis to prices $p \geq \pi_{A}$.

In the following lemma we derive the benchmark markup factor and show that benchmark revenue and auction revenue are equal.

LEMma 3 Let $p \in\left[\pi_{A}, p_{2}\right)$, let the benchmark markup factor $x_{B}\left(p, g_{1}\right)$ be the solution to (31), and denote the benchmark revenue by $\pi_{B}$. It holds that

(i) $x_{B}\left(p, g_{1}\right)=\frac{1}{g_{1}}\left(1-\frac{1-g_{1}}{\gamma_{2}(1-p)}\right)>1$.

(ii) $\pi_{B}(p)=x_{B}\left(p, g_{1}\right) \gamma_{1}(1-p) p g_{1}+p\left(1-g_{1}\right)$

(iii) $\pi_{A}$ is the unique maximizer of $\pi_{B}$. The optimal benchmark revenue is $\pi_{B}^{*}=\pi_{B}\left(\pi_{A}\right)=\pi_{A}$.

Proof See the appendix.

Part (i) of the lemma shows that the benchmark markup factor $x_{B}$ does not depend on $\sigma^{2}$. In particular, $x_{B}$ does not depend on the slope of the indirect utility function $V$. In contrast, the equilibrium markup factor $x\left(p, g_{1}\right)$ does depend on the slope of $V$ which varies in $\sigma^{2}$. In the following lemma we use the first-order condition (24) to compare the markup factors $x_{B}\left(p, g_{1}\right)$ and $x\left(p, g_{1}\right)$.

LEMMA 4 Let $p \in\left[\pi_{A}, p_{2}\right]$.

(i) If $g_{1} \in\left(0, \frac{1}{2}\right]$ the markup factor $x\left(p, g_{1}\right)$ does not exceed the benchmark markup factor $x_{B}\left(p, g_{1}\right)$.

(ii) If $g_{1} \in\left(\frac{1}{2}, 1\right)$ there exists a critical value $\sigma^{2}\left(p, g_{1}\right)>0$ of the asset's variance such that

$$
x\left(p, g_{1}\right)>x_{B}\left(p, g_{1}\right) \quad \text { or } \quad p_{e}\left(g_{1}\right)>\pi_{A} \quad \Leftrightarrow \quad \sigma^{2}<\sigma^{2}\left(p, g_{1}\right) .
$$

Proof The proof proceeds in 4 steps. 
(1) If $\sigma^{2}$ is not too small (i. e. $\left.\sigma^{2} \geq \frac{1}{8}\right) h_{p}(y)$, as defined by (21), is convex (see the appendix) and it holds by Jensen's inequality that

$$
\mathbf{E}_{g_{1}}\left[h_{p}\left(x_{B} \widetilde{Q}_{x_{B}}\right)\right] \geq h_{p}\left(\mathbf{E}_{g_{1}}\left[x_{B} \widetilde{Q}_{x_{B}}\right]\right)=h_{p}\left(x_{B} \widehat{Q}_{x_{B}}\right)=0 .
$$

Since the left hand side of this inequality is strictly increasing, we conclude that $x\left(p, g_{1}\right) \leq x_{B}\left(p, g_{1}\right)$ in this case.

(2) We take a closer look at the equilibrium equation (29) to derive conditions for $x\left(p, g_{1}\right)>x_{B}\left(p, g_{1}\right)$ and $x\left(p, g_{1}\right) \leq x_{B}\left(p, g_{1}\right)$, respectively. It is straightforward to show that $x\left(p, g_{1}\right)>x_{B}\left(p, g_{1}\right)$ or $p_{e}\left(g_{1}\right)>p$ is equivalent to

$$
\frac{x_{B}}{A_{2}^{-1}} \cdot \frac{g_{1}}{1-g_{1}}<\frac{-V^{\prime}\left(A_{2}^{-1}\right)}{V^{\prime}\left(x_{B}\right)} .
$$

Recall that $x_{B}$ does not depend on $c$. As in the preceding section we use $\Delta$, as defined by (30), to analyze the behaviour of (32).

If $\Delta>0$ it holds that the RHS of (32) is strictly increasing in $c$ with limit $\infty$ as $c$ goes to infinity. Thus, there is a number $c_{0}$ such that we have " $<"$ in (32) if and only if $c>c_{0}$ (the "only if" part follows from the fact that for small $c$ we have $\left.x\left(p, g_{1}\right) \leq x_{B}\left(p, g_{1}\right)\right)$. It follows by monotonicity that $x\left(p, g_{1}\right)>x_{B}\left(p, g_{1}\right)$ for $c>c_{0}$ and $p \geq p_{e}\left(g_{1}\right)$. This immediately implies $p_{e}\left(g_{1}\right)>\pi_{A}$ (see theorem 2).

If $\Delta \leq 0$ the RHS of (32) is decreasing in $c$ (strictly for $\Delta<0$ in which case the limit as $c$ goes to infinity is zero). By the result of step 1 we know that there is a number $\tilde{c}$ such that $x\left(p, g_{1}\right) \leq x_{B}\left(p, g_{1}\right)$ for all $c \leq \tilde{c}$. Thus, we have " $\geq$ " in (32). By monotonicity this also holds true for all $c>\tilde{c}$ which implies that $x\left(p, g_{1}\right) \leq x_{B}\left(p, g_{1}\right)$ for all $c>0$.

(3) In this step we show that $g_{1} \in\left(\frac{1}{2}, 1\right)$ corresponds to the case $\Delta>0$ while $g_{1} \in\left(0, \frac{1}{2}\right]$ corresponds to $\Delta \leq 0$. From lemma 3 we know that

$$
x_{B}-1=\frac{1}{g_{1}}-1-\frac{1-g_{1}}{g_{1}} A_{2}^{-1}=\frac{1-g_{1}}{g_{1}}\left(1-A_{2}^{-1}\right)
$$

and thus

$$
\frac{\left(x_{B}-1\right)^{2}}{\left(A_{2}^{-1}-1\right)^{2}}=\left(\frac{1-g_{1}}{g_{1}}\right)^{2}
$$


We conclude that $\Delta>0$ if and only if $g_{1}>\frac{1}{2}$.

(4) Now, we can put things together. If $g_{1} \leq \frac{1}{2}$ we have $\Delta \leq 0$. Thus, $x\left(p, g_{1}\right) \leq x_{B}\left(p, g_{1}\right)$ for all $\sigma^{2}$ in this case which proves part (i) of the lemma. Finally, if $g_{1}>\frac{1}{2}$ it holds that $\Delta>0$ in which case $x\left(p, g_{1}\right)>x_{B}\left(p, g_{1}\right)$ (or $\left.p_{e}\left(g_{1}\right)>\pi_{A}\right)$ if and only if $c>c_{0}$, where $c_{0}$ depends on $p$ and $g_{1}$. By definition of $c$ this implies that $x\left(p, g_{1}\right)>x_{B}\left(p, g_{1}\right)$ (or $\left.p_{e}\left(g_{1}\right)>\pi_{A}\right)$ is equivalent to $\sigma^{2}<c_{0}^{-1}(\mu-p)^{2}=\sigma^{2}\left(p, g_{1}\right)$. This proves part (ii) of the lemma.

From lemma 3 we know that the optimal expected benchmark revenue $\pi_{B}$ is equal to the expected auction revenue $\pi_{A}$. It follows from part (ii) of the same lemma that proportional rationing yields a lower payoff than the auction if the equilibrium markup factor $x\left(p, g_{1}\right)$ is smaller than the benchmark markup factor $x_{B}$. By lemma 4 this is the case if $g_{1} \in\left(0, \frac{1}{2}\right]$ or if $g_{1} \in\left(\frac{1}{2}, 1\right)$ and $\sigma^{2}$ is sufficiently large.

Now, suppose that $g_{1} \in\left(\frac{1}{2}, 1\right)$ and $\sigma^{2}<\sigma^{2}\left(\pi_{A}, g_{1}\right)$. Because of $x_{B}\left(\pi_{A}, g_{1}\right) \gamma_{1}\left(1-\pi_{A}\right)=1$ it follows from theorem 2 (iii) that $p_{e}\left(g_{1}\right)>\pi_{A}$. Since all shares are sold at price $p_{e}\left(g_{1}\right)$ under the fixed price mechanism, we conclude that $\pi_{F}^{*}>\pi_{A} \cdot{ }^{21}$

These surprising results are summarized in our main theorem.

\section{THEOREM 4 [Comparison of Revenues]}

(i) If $g_{1} \in\left(0, \frac{1}{2}\right]$ the revenue in the fixed price mechanism does not exceed the revenue in the auction.

(ii) If $g_{1} \in\left(\frac{1}{2}, 1\right)$ the fixed price mechanism yields a strictly higher revenue than the auction if and only if the variance of the asset is below a critical value $\sigma^{2}\left(g_{1}\right)$.

\footnotetext{
${ }^{21}$ Note that the equilibrium price in the fixed price mechanism might be larger than $p_{e}\left(g_{1}\right)$. Moreover, since the seller chooses the optimal price from the set $\left[p_{e}\left(g_{1}\right), p_{2}\right]$, the critical value of $\sigma^{2}$ depends only on $g_{1}$.
} 


\section{Concluding Remarks}

In this paper we have characterized the equilibria of a uniform price auction and a fixed price mechanism when a perfectly divisible good is sold to a large number of bidders. In addition, we have compared the expected revenues in the two mechanisms.

In the uniform price auction truthful bidding is an equilibrium. Moreover, in any pure strategy equilibrium the seller's revenue in each scenario equals the revenue from linear monopoly pricing under complete information about the demand scenario.

We have then analyzed a fixed price mechanism where the bidders are proportionally rationed in case of excess demand. We have shown that a pure strategy equilibrium of the fixed price mechanism exists. In equilibrium bidders have an incentive to overstate their demand in order to alleviate the effects of being rationed in the high demand scenario. It follows that there always exists a price that is strictly higher than the revenue in the auction when demand is low, but still yields a safe revenue (i. e. the whole quantity is sold in both scenarios). Moreover, the revenue in the fixed price mechanism is typically less volatile than the revenue in the auction. We have also shown that revealed demand depends on the variance of the asset that is offered for sale.

A comparison of the two mechanisms yields a surprising result: For certain parameter values, namely a low variance of the asset and, at the same time, a sufficiently high probability of low demand, the fixed price mechanism raises a higher expected revenue than the uniform price auction. This is rather counterintuitive, because in each scenario the uniform price auction yields the same payoff as linear monopoly prices in the case of complete information about the demand scenario. However, imperfect information allows the seller to ask for a price that is close to, or even higher, than the average price that bidders would be willing to pay in the case of complete information.

The analysis shows that a seller may benefit from demand uncertainty. 
Our results might contribute to understanding decisions of how and when to sell. Consider, for example, a monopolist who produces a good, where demand is uncertain today and is revealed in the next period. Should the firm sell before or after demand is observed? If the alternatives are to sell immediately at a fixed price or to wait until aggregate demand is known our framework applies. Using a fixed price mechanism corresponds to selling immediately while the auction yields the same revenue as selling after demand has been observed.

The ranking of the two mechanisms from the seller's point of view might not only depend on the expected revenue but also on the volatility of the payoff and on the minimum payoff (in the case of low demand). As to the first point, a risk-averse seller might prefer the fixed price mechanism even if the expected revenue is smaller than in the auction. In particular, the variance of the revenue is zero in the fixed price mechanism if the seller posts the market clearing price. Another advantage of the fixed price method, as compared to the auction, relates to the second point: Since bidders exaggerate their demand in order to avoid severe rationing in the high demand scenario, aggregate revealed demand will also be high in the low demand scenario. This enables the seller to raise a higher minimum revenue than in the auction. These issues are discussed in more detail in Bierbaum and Grimm (2002), where numerical simulations are used to provide further insights.

Our findings might provide an additional justification for the frequent use of fixed price mechanisms to allocate shares in initial public offerings, although, during the last decade auctions were proposed by many authors as an alternative. However, even in our theoretical framework that abstracts from the issue of information extraction from informed investors ${ }^{22}$ the fixed price method is not dominated by the auction in terms of revenue and has some additional benefits that might be valuable to the seller, like a less volatile payoff or a higher minimum revenue.

Still, several issues that could provide a deeper understanding of the

\footnotetext{
${ }^{22}$ This is the most common argument in favor of selling schemes that include rationing.
} 
strategic behavior in rationing games remain unsolved. First, we cannot provide a complete economic intuition that explains the way in which the asset's variance affects revealed demand. Second, it would be interesting to analyze how the model behaves if one uses other utility functions for the buyers. However, there are some technical difficulties involved. For example, the markup factor is typically not type-independent which makes it hard to show more than existence of an equilibrium and the incentive to overstate demand. Moreover, type-dependent markup factors may lead to allocations, where aftermarket trade is profitable. Finally, we have assumed that the number of bidders is large. In contrast, if the number of bidders is small each bid has an impact on the price and the allocation, which strongly affects incentives. For the uniform price auction it is well known that with a small number of bidders, who demand several units each, there are equilibria which yield a rather low revenue due to demand reduction. In the fixed price mechanism bidding less than one's true demand is still a strictly dominated strategy. However, we have not found a satisfactory characterization of the equilibria of the rationing game when a single bidder's demand has an impact on aggregate demand. These issues await further research. 


\section{Appendix}

Proof of lemma 2 We have already shown that $x_{\widetilde{Q}}$ cannot be smaller than $Q_{1}^{-1}$ or larger than $Q_{2}^{-1}$. From

$$
\left.\left(x^{2} Q_{1}^{2}-x Q_{1}\right)\right|_{x=Q_{1}^{-1}}=0 \text { and }\left.\left(x^{2} Q_{2}^{2}-x Q_{2}\right)\right|_{x=Q_{1}^{-1}}=\frac{Q_{2}}{Q_{1}}\left(\frac{Q_{2}}{Q_{1}}-1\right)<0
$$

and

$$
\left.\left(x^{2} Q_{1}^{2}-x Q_{1}\right)\right|_{x=Q_{2}^{-1}}=\frac{Q_{1}}{Q_{2}}\left(\frac{Q_{1}}{Q_{2}}-1\right)>0 \text { and }\left.\left(x^{2} Q_{2}^{2}-x Q_{2}\right)\right|_{x=Q_{2}^{-1}}=0
$$

we conclude that

$$
\left.\mathbf{E}_{g_{1}}\left[h_{p}(x \widetilde{Q})\right]\right|_{x=Q_{1}^{-1}}<0 \text { and }\left.\mathbf{E}_{g_{1}}\left[h_{p}(x \widetilde{Q})\right]\right|_{x=Q_{2}^{-1}}>0 .
$$

Since $x \mapsto \mathbf{E}_{g_{1}}\left[h_{p}(x \widetilde{Q})\right]$ is continuous we can apply the intermediate value theorem which ensures that there is a solution $x_{\widetilde{Q}} \in\left(Q_{1}^{-1}, Q_{2}^{-1}\right)$ to $(20)$. Taking the derivative with respect to $x$ we get

$$
\begin{aligned}
& \frac{\partial}{\partial x} \quad \mathbf{E}_{g_{1}}\left[h_{p}(x \widetilde{Q})\right] \\
& =\mathbf{E}_{g_{1}}\left[\left(2 x \widetilde{Q}^{2}+\widetilde{Q}+\left(x^{2} \widetilde{Q}^{2}-x \widetilde{Q}\right) c(p)\left(x \widetilde{Q}^{2}-\widetilde{Q}\right)\right) e^{c(p)\left(\frac{1}{2} x^{2} \widetilde{Q}^{2}-x \widetilde{Q}\right)}\right] \\
& =\mathbf{E}_{g_{1}}\left[\left(2 x \widetilde{Q}^{2}+\widetilde{Q}+c(p) x\left(x \widetilde{Q}^{2}-\widetilde{Q}\right)^{2}\right) e^{\left.c(p)\left(\frac{1}{2} x^{2} \widetilde{Q}^{2}-x \widetilde{Q}\right)\right]}\right. \\
& >0,
\end{aligned}
$$

where $c(p)=\left(\frac{\mu-p}{\sigma}\right)^{2}$. Thus $x_{\widetilde{Q}}$ is uniquely determined.

Proof of theorem 2 First, consider the case $g_{1} \in(0,1)$. For any given $x \geq 1$ we specify $Q_{i}$ according to condition (23) to get

$\mathbf{E}_{g_{1}}\left[h_{p}\left(x \widetilde{Q}_{x}\right)\right]= \begin{cases}h_{p}(x) g_{1}+h_{p}\left(A_{2}(p)^{-1}\right)\left(1-g_{1}\right) & \text { if } 1 \leq x<\bar{x}_{p}, \\ h_{p}\left(A_{1}(p)^{-1}\right) g_{1}+h_{p}\left(A_{2}(p)^{-1}\right)\left(1-g_{1}\right) & \text { if } x \geq \bar{x}_{p},\end{cases}$

where $\bar{x}_{p}$ is defined by

$$
\bar{x}_{p}=A_{1}(p)^{-1}
$$

To simplify notation we introduce the function

$$
\phi\left(x, p, g_{1}\right)=\mathbf{E}_{g_{1}}\left[h_{p}\left(x \widetilde{Q}_{x}\right)\right] .
$$


Taking the derivative of $\phi$ with respect to $x$ yields

$$
\frac{\partial}{\partial x} \phi\left(x, p, g_{1}\right)= \begin{cases}h_{p}^{\prime}(x) g_{1}>0 & \text { if } 1 \leq x<\bar{x}_{p} \\ 0 & \text { if } x \geq \bar{x}_{p} .\end{cases}
$$

Thus, $x \mapsto \phi\left(x, p, g_{1}\right)$ is strictly increasing on $\left[1, \bar{x}_{p}\right)$ and constant on $\left[\bar{x}_{p}, \infty\right)$. We conclude that $\phi\left(x, p, g_{1}\right)$ is maximized by $\bar{x}_{p}$. For $x=1$ we get

$$
\phi\left(1, p, g_{1}\right)=h_{p}\left(A_{2}(p)^{-1}\right)\left(1-g_{1}\right) \leq 0
$$

which holds with equality iff $p=p_{2}$. By monotonicity and the intermediate value theorem an equilibrium (in pure strategies) exists if and only if $\phi\left(\bar{x}_{p}, p, g_{1}\right) \geq 0$. We show in the sequel that there is a unique price $p_{e}=p_{e}\left(g_{1}\right)$ which solves

$$
\phi\left(\bar{x}_{p}, p, g_{1}\right)=0 \text {. }
$$

Since $\phi\left(x, p, g_{1}\right)=\phi\left(\bar{x}_{p}, p, g_{1}\right)$ for $x \geq \bar{x}_{p}$, there is a continuum of of equilibria for $p=p_{e}$, while uniqueness obtains if $\phi\left(\bar{x}_{p}, p, g_{1}\right)>0$. In the first case we have $x \geq \bar{x}_{p}=A_{1}(p)^{-1}$ which implies $D_{i}(p) \geq 1$ for $i=1,2$. In the second case it holds that $x<\bar{x}_{p}=A_{1}(p)^{-1}$ implying $D_{i}(p)<1$ for $i=1,2$. Thus, $p_{e}$ is the only market clearing price.

Since $A_{1}\left(p_{2}\right)<1$ and $A_{2}\left(p_{2}\right)=1$ it holds that $\phi\left(\bar{x}_{p_{2}}, p_{2}, g_{1}\right)>0$. A similar argument shows that $\phi\left(\bar{x}_{p_{1}}, p_{1}, g_{1}\right)<0$. By the intermediate value theorem there exists a price $p_{e} \in\left(p_{1}, p_{2}\right)$ which solves (34). To prove uniqueness of $p_{e}$ and the equilibrium markup factor $x$, it is sufficient to show that

$$
\left.\frac{\partial}{\partial p} \phi\left(\bar{x}_{p}, p, g_{1}\right)\right|_{p=p_{e}}>0 \text {. }
$$

We introduce the transforms

$$
z=\frac{1}{\mu-p} \text { and } z_{e}=\frac{1}{\mu-p_{e}} .
$$

It holds that

$$
c(p)=\left(\frac{\mu-p}{\sigma}\right)^{2}=\sigma^{-2} z^{-2}
$$

and

$$
A_{i}(p)=\gamma_{i}(\mu-p)=\frac{\gamma_{i}}{z}
$$


Inserting these identities into the RHS of (33) yields

$$
\begin{aligned}
\phi\left(\bar{x}_{p}, p, g_{1}\right) & =\mathbf{E}_{g_{1}}\left[\left(\frac{z^{2}}{\widetilde{\gamma}^{2}}-\frac{z}{\widetilde{\gamma}}\right) \exp \left(\frac{1}{\sigma^{2} z^{2}}\left(\frac{1}{2} \frac{z^{2}}{\widetilde{\gamma}^{2}}-\frac{z}{\widetilde{\gamma}}\right)\right)\right] \\
& =\mathbf{E}_{g_{1}}\left[\left(\frac{z^{2}}{\widetilde{\gamma}^{2}}-\frac{z}{\widetilde{\gamma}}\right) \exp \left(\frac{1-2 \widetilde{\gamma} z^{-1}}{2 \sigma^{2} \widetilde{\gamma}^{2}}\right)\right] \\
& =\mathbf{E}_{g_{1}}[\varphi(z, \widetilde{\gamma})],
\end{aligned}
$$

where $\widetilde{\gamma}=\gamma_{1}$ with probability $g_{1}, \widetilde{\gamma}=\gamma_{2}$ with probability $1-g_{1}$ and

$$
\varphi\left(z, \gamma_{i}\right)=\left(\frac{z^{2}}{\gamma_{i}^{2}}-\frac{z}{\gamma_{i}}\right) \exp \left(\frac{1-2 \gamma_{i} z^{-1}}{2 \sigma^{2} \gamma_{i}^{2}}\right) .
$$

Since $z^{\prime}(p)>0$ condition (35) is equivalent to

$$
\left.\frac{\partial}{\partial z} \mathbf{E}_{g_{1}}[\varphi(z, \widetilde{\gamma})]\right|_{z=z_{e}}>0
$$

Differentiating $\varphi\left(z, \gamma_{i}\right)$ with respect to $z$ yields

$$
\begin{aligned}
\frac{\partial}{\partial z} \varphi\left(z, \gamma_{i}\right)= & \left(\frac{2 z}{\gamma_{i}^{2}}-\frac{1}{\gamma_{i}}\right) \exp \left(\frac{1-\gamma_{i} z^{-1}}{2 \sigma^{2} \gamma_{i}^{2}}\right) \\
& +\left(\frac{z^{2}}{\gamma_{i}^{2}}-\frac{z}{\gamma_{i}}\right) \frac{z^{-2}}{\sigma^{2} \gamma_{i}} \exp \left(\frac{1-2 \gamma_{i} z^{-1}}{2 \sigma^{2} \gamma_{i}^{2}}\right) \\
= & \left(\frac{2 z}{\gamma_{i}^{2}}-\frac{1}{\gamma_{i}}+\frac{1}{\sigma^{2} \gamma_{i}^{2}}\left(\frac{1}{\gamma_{i}}-\frac{1}{z}\right)\right) \exp \left(\frac{1-2 \gamma_{i} z^{-1}}{2 \sigma^{2} \gamma_{i}^{2}}\right) .
\end{aligned}
$$

Now,

$$
\begin{aligned}
z \frac{\partial}{\partial z} \varphi\left(z, \gamma_{i}\right) & =\left(\frac{2 z^{2}}{\gamma_{i}^{2}}-\frac{z}{\gamma_{i}}+\frac{1}{\sigma^{2} \gamma_{i}^{2}}\left(\frac{z}{\gamma_{i}}-1\right)\right) \exp \left(\frac{1-2 \gamma_{i} z^{-1}}{2 \sigma^{2} \gamma_{i}^{2}}\right) \\
& =2 \varphi\left(z, \gamma_{i}\right)+\left(\frac{z}{\gamma_{i}}+\frac{1}{\sigma^{2} \gamma_{i}^{2}}\left(\frac{z}{\gamma_{i}}-1\right)\right) \exp \left(\frac{1-2 \gamma_{i} z^{-1}}{2 \sigma^{2} \gamma_{i}^{2}}\right)
\end{aligned}
$$

By definition of $z_{e}$ it holds that

$\left.\mathbf{E}_{g_{1}}\left[z \frac{\partial}{\partial z} \varphi(z, \widetilde{\gamma})\right]\right|_{z=z_{e}}=0+\mathbf{E}_{g_{1}}\left[\left(\frac{z_{e}}{\widetilde{\gamma}}+\frac{1}{\sigma^{2} \widetilde{\gamma}^{2}}\left(\frac{z_{e}}{\widetilde{\gamma}}-1\right)\right) \exp \left(\frac{1-2 \widetilde{\gamma} z_{e}^{-1}}{2 \sigma^{2} \widetilde{\gamma}^{2}}\right)\right]$.

Since $\gamma_{2}>z_{e}>\gamma_{1}$ we are finished if $\frac{z_{e}}{\gamma_{2}}+\frac{1}{\sigma^{2} \gamma_{2}^{2}}\left(\frac{z_{e}}{\gamma_{2}}-1\right) \geq 0$. Thus, suppose that $\frac{z_{e}}{\gamma_{2}}+\frac{1}{\sigma^{2} \gamma_{2}^{2}}\left(\frac{z_{e}}{\gamma_{2}}-1\right)<0$. In this case $(36)$ is equivalent to

$$
\frac{\frac{z_{e}}{\gamma_{1}}+\frac{1}{\sigma^{2} \gamma_{1}^{2}}\left(\frac{z_{e}}{\gamma_{1}}-1\right)}{-\left(\frac{z_{e}}{\gamma_{2}}+\frac{1}{\sigma^{2} \gamma_{2}^{2}}\left(\frac{z_{e}}{\gamma_{2}}-1\right)\right)}>\frac{\exp \left(\frac{1-2 \gamma_{2} z_{e}^{-1}}{2 \sigma^{2} \gamma_{2}^{2}}\right)\left(1-g_{1}\right)}{\exp \left(\frac{1-2 \gamma_{1} z_{e}^{-1}}{2 \sigma^{2} \gamma_{1}^{2}}\right) g_{1}} .
$$


By definition of $z_{e}$ it holds that

$$
\frac{\frac{z_{e}^{2}}{\gamma_{1}^{2}}-\frac{z_{e}}{\gamma_{1}}}{-\left(\frac{z_{e}^{2}}{\gamma_{2}^{2}}-\frac{z_{e}}{\gamma_{2}}\right)}=\frac{\exp \left(\frac{1-2 \gamma_{2} z_{e}^{-1}}{2 \sigma^{2} \gamma_{2}^{2}}\right)\left(1-g_{1}\right)}{\exp \left(\frac{1-2 \gamma_{1} z_{e}^{-1}}{2 \sigma^{2} \gamma_{1}^{2}}\right) g_{1}}
$$

Thus, we are done with this part of the proof if

$$
\frac{\frac{z_{e}}{\gamma_{1}}+\frac{1}{\sigma^{2} \gamma_{1}^{2}}\left(\frac{z_{e}}{\gamma_{1}}-1\right)}{-\left(\frac{z_{e}}{\gamma_{2}}+\frac{1}{\sigma^{2} \gamma_{2}^{2}}\left(\frac{z_{e}}{\gamma_{2}}-1\right)\right)}>\frac{\frac{z_{e}^{2}}{\gamma_{1}^{2}}-\frac{z_{e}}{\gamma_{1}}}{-\left(\frac{z_{e}^{2}}{\gamma_{2}^{2}}-\frac{z_{e}}{\gamma_{2}}\right)}
$$

or, equivalently,

$$
\frac{\gamma_{2}}{\gamma_{1}} \cdot \frac{\left(\frac{z_{e}}{\gamma_{1}}-1\right)+\sigma^{2} \gamma_{1} z_{e}}{\left(1-\frac{z_{e}}{\gamma_{2}}\right)-\sigma^{2} \gamma_{2} z_{e}}>\frac{\frac{z_{e}}{\gamma_{1}}-1}{1-\frac{z_{e}}{\gamma_{2}}}
$$

which is obviously true. This shows that $p_{e}$ is uniquely determined.

Monotonicity of $p_{e}$ in $g_{1}$ follows from the implicit function theorem:

$$
\frac{\partial}{\partial g_{1}} p_{e}\left(g_{1}\right)=-\frac{\frac{\partial}{\partial g_{1}} \phi\left(\bar{x}_{p_{e}}, p_{e}, g_{1}\right)}{\frac{\partial}{\partial p} \phi\left(\bar{x}_{p_{e}}, p_{e}, g_{1}\right)}=-\frac{h_{p_{e}}\left(A_{1}\left(p_{e}\right)^{-1}\right)-h_{p_{e}}\left(A_{2}\left(p_{e}\right)^{-1}\right)}{\left.\frac{\partial}{\partial z} \mathbf{E}_{g_{1}}[\varphi(z, \widetilde{\gamma})]\right|_{z=z_{e}} \cdot z^{\prime}\left(p_{e}\right)}<0 .
$$

Finally, it is easy to see that $p_{e}(1)=p_{1}$ and $p_{e}(0)=p_{2}$.

Proof of corollary 1 By theorem 2 we have $x\left(p, g_{1}\right) \leq A_{1}(p)^{-1}$. Therefore, revealed demand in scenario 1 satisfies

$$
D_{1}(p)=x\left(p, g_{1}\right) A_{1}(p) \leq A_{1}(p)^{-1} A_{1}(p)=1
$$

which proves the first claim.

Since $A_{2}(p) \geq 1$ for $p \leq p_{2}$ and $x\left(p, g_{1}\right)>1$ by lemma 2 revealed demand in scenario 2 satisfies

$$
D_{2}(p)=x\left(p, g_{1}\right) A_{2}(p)>1 \text {. }
$$

It follows that supply to buyer $\theta$ is given by

$$
x\left(p, g_{1}\right) \alpha(p, \theta) Q_{2}(p)=\frac{x\left(p, g_{1}\right) \alpha(p, \theta)}{x\left(p, g_{1}\right) A_{2}(p)}=\frac{\alpha(p, \theta)}{A_{2}(p)} \leq \alpha(p, \theta),
$$

where equality holds if and only if $p=p_{2}$. 
Appendix to the proof of theorem 3 First note that if the upper limit is not binding, an equilibrium of the bidding stage exists at all prices $p \in$ $\left[p_{e}\left(g_{1}\right), p_{2}\right]$. Now suppose that at a price $p$ a bidder $\theta$ 's revealed demand is capped by $\bar{d}$ so that he cannot choose his best reply rationing factor $x_{\widetilde{Q}}\left(p, g_{1}\right)$ since this implied $x_{\widetilde{Q}}\left(p, g_{1}\right) \alpha(p, \theta)>\bar{d}$. However, since the marginal utility from increasing $x$ is positive ${ }^{23}$ for all $x \in\left[1, x_{\widetilde{Q}}\left(p, g_{1}\right)\right)$, the expected utility of a bidder who is constrained by an upper limit on revealed demand is maximized by choosing the upper limit. Therefore the optimal markup is given by $x_{\theta}\left(p, g_{1}\right)=\min \left(x_{\widetilde{Q}}\left(p, g_{1}\right), \frac{\bar{d}}{\alpha(p, \theta)}\right)$, so that

$$
\begin{aligned}
d\left(p, g_{1}, \theta\right) & =x_{\theta}\left(p, g_{1}\right) \alpha(p, \theta) \\
& =\min \left\{x_{\widetilde{Q}}\left(p, g_{1}\right) \alpha(p, \theta), \bar{d}\right\} .
\end{aligned}
$$

This implies that the rationing factor $\widetilde{Q}_{x}$ faced by the bidders in equilibrium is given by its realizations

$$
Q_{i}=\min \left(1, \frac{1}{\int_{\underline{\theta}}^{\bar{\theta}} x_{\theta}\left(p, g_{1}\right) \alpha(p, \theta) d F_{i}(\theta)}\right) .
$$

Note that due to the upper limit on revealed demand an equilibrium of the bidding stage exists at all prices in $\left[p_{1}, p_{2}\right]$, however, whenever the upper limit is binding for one or more bidders, the type independence of the equilibrium markup factor no longer holds.

Proof of lemma 3 If a buyer is risk-neutral with respect to rationing (the choice of $x$ ), he maximizes utility given the expected value $\hat{Q}_{x}=\mathbf{E}_{g_{1}}\left[\widetilde{Q}_{x}\right]$ of $\widetilde{Q}_{x}$. From the FOC $(20)$ it follows that $x \hat{Q}_{x}=1$. Since $\hat{Q}_{x} \leq 1$ it holds that $x \geq 1$. By definition of $\widetilde{Q}_{x}$ and $A_{2} \geq 1$ we get

$$
\begin{aligned}
1 & =x \widehat{Q}_{x}=x \min \left(1, \frac{1}{x A_{1}}\right) g_{1}+x \min \left(1, \frac{1}{x A_{2}}\right)\left(1-g_{1}\right) \\
& =x \min \left(1, \frac{1}{x A_{1}}\right) g_{1}+\frac{1}{A_{2}}\left(1-g_{1}\right) .
\end{aligned}
$$

${ }^{23}$ This follows from the monotonicity of $E_{g_{1}}\left[h_{p}(x \widetilde{Q})\right]$, as shown in the proof of lemma 2 . 
It is easy to show that a number $x$ which has $x A_{1}>1$ cannot be a solution to this equation. Therefore, we assume that $x A_{1} \leq 1$ to get $^{24}$ the following condition:

$$
1=x g_{1}+\frac{1}{A_{2}}\left(1-g_{1}\right) .
$$

Solving for $x$ yields (recall that $A_{i}=\gamma_{i}(1-p)$ )

$$
x_{B}\left(p, g_{1}\right)=\frac{1}{g_{1}}\left(1-\frac{1-g_{1}}{\gamma_{2}(1-p)}\right) .
$$

Thus, revealed demand in scenario 1 is given by

$$
\begin{aligned}
x_{B}\left(p, g_{1}\right) \gamma_{1}(1-p) & =\frac{1}{g_{1}}\left(1-\frac{1-g_{1}}{\gamma_{2}(1-p)}\right) \gamma_{1}(1-p) \\
& =\gamma_{1} \frac{1}{g_{1}}(1-p)-\frac{\gamma_{1}}{\gamma_{2}} \frac{1-g_{1}}{g_{1}}
\end{aligned}
$$

and its value at $\pi_{A}=1-\frac{g_{1}}{\gamma_{1}}-\frac{1-g_{1}}{\gamma_{2}}$ is

$$
x_{B}\left(\pi_{A}\right) \gamma_{1}\left(1-\pi_{A}\right)=\gamma_{1} \frac{1}{g_{1}}\left(\frac{g_{1}}{\gamma_{1}}+\frac{1-g_{1}}{\gamma_{2}}\right)-\frac{\gamma_{1}}{\gamma_{2}} \frac{1-g_{1}}{g_{1}}=1 .
$$

The benchmark revenue at $p \in\left[\pi_{A}, p_{2}\right]$ is

$$
\begin{aligned}
\pi_{B}(p) & =x_{B}(p) \gamma_{1}(1-p) p g_{1}+p\left(1-g_{1}\right) \\
& =\gamma_{1}(1-p) p+\left(1-\frac{\gamma_{1}}{\gamma_{2}}\right)\left(1-g_{1}\right) p
\end{aligned}
$$

and the derivative of $\pi_{B}$,

$$
\frac{\partial}{\partial p} \pi_{B}(p)=\gamma_{1}(1-2 p)+\left(1-\frac{\gamma_{1}}{\gamma_{2}}\right)\left(1-g_{1}\right),
$$

is obviously decreasing in $p$. Since $\gamma_{1} \geq 2$ and $\gamma_{2}>\gamma_{1}$ it holds that

$$
\begin{aligned}
\frac{\partial}{\partial p} \pi_{B}\left(\pi_{A}\right) & =\gamma_{1}\left(1-2+\frac{2 g_{1}}{\gamma_{1}}+\frac{2\left(1-g_{1}\right)}{\gamma_{2}}\right)+\left(1-\frac{\gamma_{1}}{\gamma_{2}}\right)\left(1-g_{1}\right) \\
& =-\gamma_{1}+2 g_{1}+\frac{\gamma_{1}\left(1-g_{1}\right)}{\gamma_{2}}+\left(1-g_{1}\right) \\
& <2 g_{1}+2\left(1-g_{1}\right)-\gamma_{1} \\
& =2-\gamma_{1} \\
& \leq 0 .
\end{aligned}
$$

\footnotetext{
${ }^{24}$ We show later, that the solution $x_{B}$ to this equation satisfies $x_{B} A_{1} \leq 1$ as long as $p \geq \pi_{A}$.
} 
Thus, marginal profit is negative at $p=\pi_{A}$. This also holds for all $p \in\left[\pi_{A}, p_{2}\right]$, since marginal profit, as given by (38), is decreasing. Since revealed demand at $p=\pi_{A}$ is equal to one and profit is decreasing in $p$ for $p \in\left[\pi_{A}, p_{2}\right]$, the benchmark case yields the same expected profit as the auction, which proves part (b) of the lemma.

Proof of lemma 4 Proof that $h_{p}(y)$ is convex if $\sigma^{2}$ is large enough:

Now, we show that $h_{p}$ is convex if $c$ is small enough. The derivatives are

$$
h_{p}^{\prime}(y)=\left(2 y-1+c\left(y^{2}-y\right)(y-1)\right) \exp \left(c\left(\frac{1}{2} y^{2}-y\right)\right)
$$

and

$$
h_{p}^{\prime \prime}(y)=\left(2+2(2 y-1) c(y-1)+\left(y^{2}-y\right)\left(c+c^{2}(y-1)^{2}\right)\right) e^{c\left(\frac{1}{2} y^{2}-y\right)} .
$$

Note that

$$
\begin{aligned}
h_{p}^{\prime \prime}(y) & >\left(2+2 c\left(2 \frac{3}{4}-1\right)\left(\frac{3}{4}-1\right)+\left(\frac{1}{4}-\frac{1}{2}\right)\left(c+c^{2}\right)\right) \exp \left(c\left(\frac{1}{2} y^{2}-y\right)\right) \\
& =\left(2-\frac{1}{4} c-\frac{1}{4}\left(c+c^{2}\right)\right) \exp \left(c\left(\frac{1}{2} y^{2}-y\right)\right) \\
& =\left(2-\frac{1}{2} c-\frac{1}{4} c^{2}\right) \exp \left(c\left(\frac{1}{2} y^{2}-y\right)\right) .
\end{aligned}
$$

It follows that $c \leq 2$ is sufficient for $h_{p}$ being convex. Since $(1-p)^{2} \leq \frac{1}{4}$ for $p \geq p_{1}$ this is always the case if $\sigma^{2} \geq \frac{1}{8}$.

\section{References}

Back, K., and J. P. Zender (1993). Auctions of Divisible Goods: On the Rationale for the Treasury Experiment. Review of Financial Studies 6 (4), pp. 733-764.

Benveniste, L. M. and W. J. Wilhelm (1990). A Comparative Analysis of IPO Proceeds under Alternative Regulatory Environments. Journal of Financial Economics 28, pp. 173-207. 
Biais, B. and A. M. Faugeron-Crouzet (2000). IPO Auctions: English, Dutch, ..., French and Internet. Working Paper.

Bierbaum, J., and V. Grimm (2002). Uniform Price Auctions vs. Fixed Price Mechanisms: A Numerical Analysis. Working Paper.

Blonski, M. (2001). Equilibrium Characterization in Large Anonymous Games. Working Paper.

Bulow, J. and P. Klemperer (1997). The Winner's Curse and the Failure of the Law of Demand, Research Paper No. 1465, Graduate School of Business, Stanford University.

Fudenberg, D. and J. Tirole (1998). Game Theory. MIT Press, Cambridge, Massachusetts.

Gilbert, R. and P. Klemperer (2000). An Equilibrium Theory of Rationing. RAND Journal of Economics 31 (1), pp. $1-21$.

Herrero, C. and A. Villar (2001). The Three Musketeers. Four Classical Solutions to Bankruptcy Problems. Mathematical Social Sciences, 42, pp. $307-328$

Kandel, S., Sarig, O., and A. Wohl (1999). The Demand for Stocks: An Analysis of IPO Auctions. Review of Financial Studies 12 (2), pp. $227-247$.

Moulin, H. (2000). Priority Rules and Other Asymmetric Rationing Methods. Econometrica 68 (3), pp. 643-684.

Mas-Colell, A., M. D. Whinston, and J. R. Green (1995). Microeconomic Theory, Oxford University Press, New York, Oxford.

Nautz, D. (1995). Optimal Bidding in Multi-Unit Auctions with Many Bidders. Economics Letters 48 (3), pp. 301-306. 
Nautz, D., and J. Oechssler (2003). The Repo Auctions of the European Central Bank and the Vanishing Quota Puzzle. Scandinavian Journal of Economics 105, pp. 207-220.

Nautz, D., and E. Wolfstetter (1997). Bid Shading and Risk Aversion in Multi-Unit Auctions with many Bidders. Economics Letters 56 (2), pp. 195-200.

Sherman, A. E. (2000a). Global Trends in IPO Methods: Book Building vs. Auctions. Working Paper, University of Minnesota.

Sherman, A. E. (2000b). IPOs and Long-Term Relationships: An Advantage of Book Building. Review of Financial Studies, 13 (3), pp. $697-714$.

Sherman, A. E. and S. Titman (2000). Building the IPO Order Book: Underpricing and Participation Limits with Costly Information. NBER Working Paper No. 7786, Cambridge, MA.

Swinkels, J. M. (2001). Efficiency of Large Private Value Auctions. Econometrica 69 (1), pp. 37-68.

Wilson, R. (1979). Auctions of Shares. Quaterly Journal of Economics 93, pp. 675-698. 